\title{
Boundary value problems for Hilfer type sequential fractional differential equations and inclusions involving Riemann-Stieltjes integral multi-strip boundary conditions
}

\author{
Cholticha Nuchpong ${ }^{1}$, Sotiris K. Ntouyas ${ }^{2,3}$, Ayub Samadi ${ }^{4}$ and Jessada Tariboon ${ }^{5^{*}}$ (D)
}

${ }^{*}$ Correspondence:

jessada.t@sci.kmutnb.ac.th

Intelligent and Nonlinear Dynamic

Innovations Research Center,

Department of Mathematics,

Faculty of Applied Science, King

Mongkut's University of Technology

North Bangkok, Bangkok 10800,

Thailand

Full list of author information is

available at the end of the article

\begin{abstract}
In this paper, we study boundary value problems for sequential fractional differential equations and inclusions involving Hilfer fractional derivatives, supplemented with Riemann-Stieltjes integral multi-strip boundary conditions. Existence and uniqueness results are obtained in the single-valued case by using the classical Banach and Krasnosel'skii fixed point theorems and the Leray-Schauder nonlinear alternative. In the multi-valued case an existence result is proved by using nonlinear alternative for contractive maps. Examples illustrating our results are also presented.
\end{abstract}

MSC: 26A33; 34A08; 34A60; 34B15

Keywords: Fractional differential equations; Fractional differential inclusions; Hilfer fractional derivative; Riemann-Liouville fractional derivative; Caputo fractional derivative; Boundary value problems; Existence and uniqueness; Fixed point theory

\section{Introduction and preliminaries}

Differential equations of fractional order describe many real world processes more accurately as compared to classical order differential equations. For this reason differential equations of fractional order with initial/boundary conditions have been studied by many researchers. Fractional differential equations arise in lots of engineering and clinical disciplines which include biology, physics, chemistry, economics, signal and image processing, control theory, and so on; see the monographs [1-8].

Several different definitions of fractional integrals and derivatives exist in the literature. The most popular ones are the Riemann-Liouville fractional derivative of order $\alpha>0$ defined for a continuous function by

$$
{ }^{R L} D^{\alpha} u(t):=D^{n} I^{n-\alpha} u(t)=\frac{1}{\Gamma(n-\alpha)}\left(\frac{d}{d t}\right)^{n} \int_{a}^{t}(t-s)^{n-\alpha-1} u(s) d s, \quad n-1<\alpha<n,
$$

(c) The Author(s) 2021. This article is licensed under a Creative Commons Attribution 4.0 International License, which permits use, sharing, adaptation, distribution and reproduction in any medium or format, as long as you give appropriate credit to the original author(s) and the source, provide a link to the Creative Commons licence, and indicate if changes were made. The images or other third party material in this article are included in the article's Creative Commons licence, unless indicated otherwise in a credit line to the material. If material is not included in the article's Creative Commons licence and your intended use is not permitted by statutory regulation or exceeds the permitted use, you will need to obtain permission directly from the copyright holder. To view a copy of this licence, visit http://creativecommons.org/licenses/by/4.0/. 
and the Caputo fractional derivative of order $\alpha>0$ defined by

$$
{ }^{C} D^{\alpha} u(t):=I^{n-\alpha} D^{n} u(t)=\frac{1}{\Gamma(n-\alpha)} \int_{a}^{t}(t-s)^{n-\alpha-1}\left(\frac{d}{d s}\right)^{n} u(s) d s, \quad n-1<\alpha<n .
$$

In the above definitions $I^{(\cdot)}$ is the Riemann-Liouville fractional integral of order $\alpha>0$ which is defined by

$$
I^{\alpha} u(t)=\frac{1}{\Gamma(\alpha)} \int_{a}^{t}(t-s)^{\alpha-1} u(s) d s, \quad n-1<\alpha<n,
$$

provided the right-hand side is point-wise defined on $(a, \infty)$. Other less-known definitions of fractional integrals and derivatives are the Hadamard fractional derivative, the Erdélyi-Kober fractional derivative, and so on. Hilfer in [9] generalized both RiemannLiouville and Caputo fractional derivatives. This derivative, known as the Hilfer fractional derivative, is defined by

$$
{ }^{H} D^{\alpha, \beta} u(t)=I^{\beta(n-\alpha)} D^{n} I^{(1-\beta)(n-\alpha)} u(t),
$$

where $n-1<\alpha<n, 0 \leq \beta \leq 1, t>a, D=\frac{d}{d t}$. Fractional differential equations involving Hilfer derivative have many applications, we refer to [10] and the references cited therein. There are actual world occurrences with uncharacteristic dynamics such as atmospheric diffusion of pollution, signals transmissions through strong magnetic fields, the effect of the theory of the profitability of stocks in economic markets, the theoretical simulation of dielectric relaxation in glass forming materials, network traffic, and so on. See [11, 12] and the references cited therein. We refer to $[13,14]$ and the references cited therein for some properties of the Hilfer derivative, and to [15-17] for initial value problems involving Hilfer fractional derivatives.

In contrast to classical boundary conditions, nonlocal conditions help to formulate the changes happening on certain positions and segments of the given domain [18]. On the other hand, boundary conditions involving derivatives and integrals provide a decent approach to describe non-uniformities on segments of curved boundary structures. Examples include fluid problems [19], biomedical applications [20], engineering applications $[21,22]$, etc.

In [23] the authors initiated the study of nonlocal boundary value problems for Hilfer fractional derivative by studying the boundary value problem of Hilfer-type fractional differential equations with nonlocal integral boundary conditions

$$
\begin{aligned}
& { }^{H} \mathfrak{D}^{\alpha, \beta} x(t)=f(t, x(t)), \quad t \in[a, b], 1<\alpha<2,0 \leq \beta \leq 1, \\
& x(a)=0, \quad x(b)=\sum_{i=1}^{m} \delta_{i} \mathcal{I}^{\varphi_{i}} x\left(\xi_{i}\right), \quad \varphi_{i}>0, \delta_{i} \in \mathbb{R}, \xi_{i} \in[a, b],
\end{aligned}
$$

where ${ }^{H} \mathfrak{D}^{\alpha, \beta}$ is the Hilfer fractional derivative of order $\alpha, 1<\alpha<2$, and parameter $\beta$, $0 \leq \beta \leq 1, \mathcal{I}^{\varphi_{i}}$ is the Riemann-Liouville fractional integral of order $\varphi_{i}>0, \xi_{i} \in[a, b], a \geq 0$, and $\delta_{i} \in \mathbb{R}$. Several existence and uniqueness results were proved by using a variety of fixed point theorems. 
In a series of papers [7-27] nonlocal boundary value problems involving Hilfer fractional derivatives were studied with a variety of boundary conditions. Thus, in [7] the authors studied Hilfer-Langevin three-point fractional boundary value problems, in [24] pantograph Hilfer fractional boundary value problems with nonlocal integral boundary conditions were studied, in [25] Hilfer fractional boundary value problems with nonlocal integral integro-multipoint boundary conditions were studied, in [26] Hilfer fractional boundary value problems with nonlocal multipoint, fractional derivative multi-order, and fractional integral multi-order boundary conditions were studied, and in [27] sequential Hilfer fractional boundary value problems with nonlocal integro-multipoint boundary conditions were studied.

In the present paper, motivated by the recent interest in studying boundary value problems involving Hilfer fractional derivative operators, we discuss a new class of sequential Hilfer-type boundary value problems for fractional differential equations involving Riemann-Stieltjes integral multi-strip boundary conditions of the form

$$
\left\{\begin{array}{l}
\left({ }^{H} D^{\alpha, \beta}+k^{H} D^{\alpha-1, \beta}\right) x(t)=f(t, x(t)), \quad t \in[a, b], \\
x(a)=0, \quad x(b)=\lambda \int_{a}^{b} x(s) d H(s)+\sum_{i=1}^{n} \mu_{i} \int_{\eta_{i}}^{\xi_{i}} x(s) d s,
\end{array}\right.
$$

where ${ }^{H} D^{\alpha, \beta}$ denotes the Hilfer fractional derivative operator of order $\alpha, 1<\alpha<2$, and parameter $\beta, 0 \leq \beta \leq 1, f:[a, b] \times \mathbb{R} \rightarrow \mathbb{R}$ is a continuous function, $\int_{a}^{b} x(s) d H(s)$ is the Riemann-Stieltjes integral with respect to the function $H:[a, b] \rightarrow \mathbb{R}, a \geq 0, k, \mu_{i} \in \mathbb{R}$, $a<\eta_{i}<\xi_{i} \leq b, i=1,2, \ldots, n$.

We establish existence and uniqueness results by using the basic tools from fixed point theory. The existence of a unique solution is proved by using the Banach contraction mapping principle, while in the two existence results we make use of the Krasnosel'skii fixed point theorem and the nonlinear alternative of Leray-Schauder type. The main results are presented in Sect. 2. The obtained results are well illustrated by numerical examples. The results obtained in the present paper are new and significantly contribute to the existing literature on the topic.

We also cover the multi-valued case of problem (1.3) by considering the following sequential inclusion boundary value problem:

$$
\left\{\begin{array}{l}
\left({ }^{H} D^{\alpha, \beta}+k^{H} D^{\alpha-1, \beta}\right) x(t) \in F(t, x(t)), \quad t \in[a, b], \\
x(a)=0, \quad x(b)=\lambda \int_{a}^{b} x(s) d H(s)+\sum_{i=1}^{n} \mu_{i} \int_{\eta_{i}}^{\xi_{i}} x(s) d s,
\end{array}\right.
$$

where $F:[a, b] \times \mathbb{R} \rightarrow \mathcal{P}(\mathbb{R})$ is a multi-valued function, $(\mathcal{P}(\mathbb{R})$ is the family of all nonempty subsets of $\mathbb{R})$.

An existence result is proved in Sect. 3 for the sequential Hilfer inclusion boundary value problem (1.4) via nonlinear alternative for contractive maps.

\section{Existence and uniqueness results for problem (1.3)}

The following auxiliary lemma, concerning a linear variant of the boundary value problem (1.3), plays a fundamental role in establishing the existence and uniqueness results for the given nonlinear problem. 
Lemma 2.1 ([14]) Let $f \in L(a, b), n-1<\alpha \leq n, n \in \mathbb{N}, 0 \leq \beta \leq 1, I^{(n-\alpha)(1-\beta)} f \in A C^{k}[a, b]$. Then

$$
\left(I^{\alpha H} D^{\alpha, \beta} f\right)(t)=f(t)-\sum_{k=0}^{n-1} \frac{(t-a)^{k-(n-\alpha)(1-\beta)}}{\Gamma(k-(n-\alpha)(1-\beta)+1)} \lim _{t \rightarrow a^{+}}\left(I^{(1-\beta)(n-\alpha)} f\right)(t) .
$$

Lemma 2.2 Let $a \geq 0,1<\alpha<2, \gamma=\alpha+2 \beta-\alpha \beta, h \in C([a, b], \mathbb{R})$, and

$$
\Lambda:=(b-a)^{\gamma-1}-\lambda \int_{a}^{b}(s-a)^{\gamma-1} d H(s)-\frac{1}{\gamma} \sum_{i=1}^{n} \mu_{i}\left[\left(\xi_{i}-a\right)^{\gamma}-\left(\eta_{i}-a\right)^{\gamma}\right] \neq 0 .
$$

Then the function $x$ is a solution of the sequential boundary value problem

$$
\left\{\begin{array}{l}
\left({ }^{H} D^{\alpha, \beta}+k^{H} D^{\alpha-1, \beta}\right) x(t)=h(t), \quad t \in[a, b], 1<\alpha<2,0 \leq \beta \leq 1, \\
x(a)=0, \quad x(b)=\lambda \int_{a}^{b} x(s) d H(s)+\sum_{i=1}^{n} \mu_{i} \int_{\eta_{i}}^{\xi_{i}} x(s) d s,
\end{array}\right.
$$

if and only if

$$
\begin{aligned}
x(t)= & I^{\alpha} h(t)-k \int_{a}^{t} x(s) d s+\frac{(t-a)^{\gamma-1}}{\Lambda}\left[-\lambda \int_{a}^{b}\left[k \int_{a}^{s} x(u) d u-I^{\alpha} h(s)\right] d H(s)\right. \\
& -k \sum_{i=1}^{n} \mu_{i} \int_{\eta_{i}}^{\xi_{i}} \int_{a}^{s} x(u) d u d s+\sum_{i=1}^{n} \mu_{i} \int_{\eta_{i}}^{\xi_{i}} I^{\alpha} h(s) d s \\
& \left.+k \int_{a}^{b} x(s) d s-I^{\alpha} h(b)\right] .
\end{aligned}
$$

Proof Let $x$ be a solution of the nonlocal sequential boundary value problem (2.2). By Lemma 2.1, applying on both sides of equation (2.2) the fractional integral $I^{\alpha}$, we have

$$
\begin{aligned}
x(t) & =c_{0} \frac{(t-a)^{-(2-\alpha)(1-\beta)}}{\Gamma(1-(2-\alpha)(1-\beta))}+c_{1} \frac{(t-a)^{1-(2-\alpha)(1-\beta)}}{\Gamma(2-(2-\alpha)(1-\beta))}-k I^{1} x(t)+I^{\alpha} h(t) \\
& =c_{0} \frac{(t-a)^{\gamma-2}}{\Gamma(\gamma-1)}+c_{1} \frac{(t-a)^{\gamma-1}}{\Gamma(\gamma)}-k I^{1} x(t)+I^{\alpha} h(t),
\end{aligned}
$$

since $(1-\beta)(2-\alpha)=2-\gamma$, where $c_{0}$ and $c_{1}$ are arbitrary real constants.

From $x(a)=0$ we have $c_{0}=0$. Then we get

$$
x(t)=c_{1} \frac{(t-a)^{\gamma-1}}{\Gamma(\gamma)}-k I^{1} x(t)+I^{\alpha} h(t)
$$

From the boundary condition $x(b)=\lambda \int_{a}^{b} x(s) d H(s)+\sum_{i=1}^{n} \mu_{i} \int_{\eta_{i}}^{\xi_{i}} x(s) d s$, we found

$$
\begin{aligned}
c_{1}= & \frac{\Gamma(\gamma)}{\Lambda}\left[-\lambda \int_{a}^{b}\left[k \int_{a}^{s} x(u) d u-I^{\alpha} h(s)\right] d H(s)\right. \\
& \left.-k \sum_{i=1}^{n} \mu_{i} \int_{\eta_{i}}^{\xi_{i}} \int_{a}^{s} x(u) d u d s+\sum_{i=1}^{n} \mu_{i} \int_{\eta_{i}}^{\xi_{i}} I^{\alpha} h(s) d s+k \int_{a}^{b} x(s) d s-I^{\alpha} h(b)\right] .
\end{aligned}
$$


Substituting the value of $c_{1}$ in (2.4), we obtain solution (2.3). The converse can be proven by direct computation. The proof is finished.

We denote by $C([a, b], \mathbb{R})$ the Banach space of all continuous functions from $[a, b]$ to $\mathbb{R}$ with the norm $\|x\|=\sup _{t \in[a, b]}|x(t)|$. In view of Lemma 2.2, we define an operator $\mathcal{A}$ : $C([a, b], \mathbb{R}) \rightarrow C([a, b], \mathbb{R})$ by

$$
\begin{aligned}
(\mathcal{A} x)(t)= & I^{\alpha} f(t, x(t))-k \int_{a}^{t} x(s) d s \\
& +\frac{(t-a)^{\gamma-1}}{\Lambda}\left[-\lambda \int_{a}^{b}\left[k \int_{a}^{s} x(u) d u-I^{\alpha} f(s, x(s))\right] d H(s)\right. \\
& -k \sum_{i=1}^{n} \mu_{i} \int_{\eta_{i}}^{\xi_{i}} \int_{a}^{s} x(u) d u d s+\sum_{i=1}^{n} \mu_{i} \int_{\eta_{i}}^{\xi_{i}} I^{\alpha} f(s, x(s)) d s \\
& \left.+k \int_{a}^{b} x(s) d s-I^{\alpha} f(b, x(b))\right] .
\end{aligned}
$$

It is obvious that the sequential boundary value problem (1.3) has solutions if and only if the operator $\mathcal{A}$ has fixed points.

For computational convenience we use the notations:

$$
\begin{aligned}
\Omega= & \frac{(b-a)^{\alpha}}{\Gamma(\alpha+1)}+\frac{(b-a)^{\gamma-1}}{|\Lambda|}\left[|\lambda| \int_{a}^{b} \frac{(s-a)^{\alpha}}{\Gamma(\alpha+1)} d H(s)\right. \\
& \left.+\sum_{i=1}^{n}\left|\mu_{i}\right| \frac{\left(\xi_{i}-a\right)^{\alpha+1}-\left(\eta_{i}-a\right)^{\alpha+1}}{\Gamma(\alpha+2)}+\frac{(b-a)^{\alpha}}{\Gamma(\alpha+1)}\right],
\end{aligned}
$$

and

$$
\begin{aligned}
\Omega_{1}= & |k|(b-a)+\frac{(b-a)^{\gamma-1}}{|\Lambda|}\left[|\lambda||k| \int_{a}^{b}(s-a) d H(s)\right. \\
& \left.+\frac{1}{2}|k| \sum_{i=1}^{n}\left|\mu_{i}\right|\left(\left(\xi_{i}-a\right)^{2}-\left(\eta_{i}-a\right)^{2}\right)+|k|(b-a)\right] .
\end{aligned}
$$

By using well-known fixed point theorems, we prove existence, as well as existence and uniqueness results, for the sequential Hilfer-type boundary value problem (1.3) in the following sections.

\subsection{Existence and uniqueness result}

In our first result we prove the existence of a unique solution for the sequential Hilfer boundary value problem (1.3) via Banach's contraction mapping principle.

Theorem 2.1 Assume that:

$\left(H_{1}\right)|f(t, x)-f(t, y)| \leq L|x-y|, L>0, t \in[a, b]$, and $x, y \in \mathbb{R}$.

If

$$
L \Omega+\Omega_{1}<1,
$$


where $\Omega$ and $\Omega_{1}$ are defined by (2.6) and (2.7) respectively, then the sequential Hilfer boundary value problem (1.3) has a unique solution on $[a, b]$.

Proof With the help of the operator $\mathcal{A}$ defined as in (2.5), we transform the sequential Hilfer boundary value problem (1.3) into a fixed point problem, $x=\mathcal{A} x$. We shall show that the operator $\mathcal{A}$ has a unique fixed point by applying the Banach contraction mapping principle.

Set $\sup _{t \in[a, b]}|f(t, 0)|=M<\infty$, and choose $r>0$ such that

$$
r \geq \frac{M \Omega}{1-L \Omega-\Omega_{1}}
$$

Let $B_{r}=\{x \in C([a, b], \mathbb{R}):\|x\| \leq r\}$. We show that $\mathcal{A} B_{r} \subset B_{r}$.

For any $x \in B_{r}$, we have

$$
\begin{aligned}
|(\mathcal{A} x)(t)| \leq & I^{\alpha}|f(t, x(t))|+|k| \int_{a}^{t}|x(s)| d s \\
& +\frac{(b-a)^{\gamma-1}}{|\Lambda|}\left[|\lambda| \int_{a}^{b}\left[|k| \int_{a}^{s}|x(u)| d u+I^{\alpha}|f(s, x(s))|\right] d H(s)\right. \\
& +|k| \sum_{i=1}^{n}\left|\mu_{i}\right| \int_{\eta_{i}}^{\xi_{i}} \int_{a}^{s}|x(u)| d u d s+\sum_{i=1}^{n}\left|\mu_{i}\right| \int_{\eta_{i}}^{\xi_{i}} I^{\alpha}|f(s, x(s))| d s \\
& \left.+|k| \int_{a}^{b}|x(s)| d s+I^{\alpha}|f(b, x(b))|\right] \\
\leq & I^{\alpha}(|f(t, x(t))-f(t, 0)|+|f(t, 0)|)+|k| \int_{a}^{t}|x(s)| d s \\
& +\frac{(b-a)^{\gamma-1}}{|\Lambda|}\left[| \lambda | \int _ { a } ^ { b } \left[|k| \int_{a}^{s}|x(u)| d u+I^{\alpha}(|f(s, x(s))-f(s, 0)|\right.\right. \\
& +|f(s, 0)|)] d H(s)+|k| \sum_{i=1}^{n}\left|\mu_{i}\right| \int_{\eta_{i}}^{\xi_{i}} \int_{a}^{s}|x(u)| d u d s \\
& +\sum_{i=1}^{n}\left|\mu_{i}\right| \int_{\eta_{i}}^{\xi_{i}} I^{\alpha}(|f(s, x(s))-f(s, 0)|+|f(s, 0)|) d s \\
& \left.+|k| \int_{a}^{b}|x(s)| d s+I^{\alpha}(|f(b, x(b))-f(b, 0)|+|f(b, 0)|)\right] \\
\leq & \left\{\frac{(b-a)^{\alpha}}{\Gamma(\alpha+1)}+\frac{(b-a)^{\gamma-1}}{|\Lambda|}\left[|\lambda| \int_{a}^{b} \frac{(s-a)^{\alpha}}{\Gamma(\alpha+1)} d H(s)\right.\right. \\
& \left.\left.+\sum_{i=1}^{n}\left|\mu_{i}\right| \frac{\left(\xi_{i}-a\right)^{\alpha+1}-\left(\eta_{i}-a\right)^{\alpha+1}}{\Gamma(\alpha+2)}+\frac{(b-a)^{\alpha}}{\Gamma(\alpha+1)}\right]\right\}(L\|x\|+M) \\
& \left\{k \mid(b-a)+\frac{(b-a)^{\gamma-1}}{|\Lambda|}\left[|\lambda||k| \int_{a}^{b}(s-a) d H(s)\right.\right. \\
& \\
& (\alpha) \\
&
\end{aligned}
$$




$$
\begin{aligned}
& \left.\left.\quad+\frac{1}{2}|k| \sum_{i=1}^{n}\left|\mu_{i}\right|\left(\left(\xi_{i}-a\right)^{2}-\left(\eta_{i}-a\right)^{2}\right)+|k|(b-a)\right]\right\}\|x\| \\
& \leq(L r+M) \Omega+\Omega_{1} r \leq r,
\end{aligned}
$$

and consequently $\|\mathcal{A} x\| \leq r$, which implies that $\mathcal{A} B_{r} \subset B_{r}$.

Next, we show that $\mathcal{A}$ is a contraction. Let $x, y \in C([a, b], \mathbb{R})$. We have, for $t \in[a, b]$,

$$
\begin{aligned}
|(\mathcal{A} x)(t)-(\mathcal{A} y)(t)| & \\
\leq & I^{\alpha}|f(t, x(t))-f(t, y(t))|+|k| \int_{a}^{t}|x(s)-y(s)| d s \\
& +\frac{(b-a)^{\gamma-1}}{|\Lambda|}\left[|\lambda| \int_{a}^{b}\left[|k| \int_{a}^{s}|x(u)-y(u)| d u+I^{\alpha}|f(s, x(s))-f(s, y(s))|\right] d H(s)\right. \\
& +|k| \sum_{i=1}^{n}\left|\mu_{i}\right| \int_{\eta_{i}}^{\xi_{i}} \int_{a}^{s}|x(u)-y(u)| d u d s+\sum_{i=1}^{n}\left|\mu_{i}\right| \int_{\eta_{i}}^{\xi_{i}} I^{\alpha}|f(s, x(s))-f(s, y(s))| d s \\
& \left.+|k| \int_{a}^{b}|x(s)-y(s)| d s+I^{\alpha}|f(b, x(b))-f(b, y(b))|\right] \\
\leq & \left\{\frac{(b-a)^{\alpha}}{\Gamma(\alpha+1)}+\frac{(b-a)^{\gamma-1}}{|\Lambda|}\left[|\lambda| \int_{a}^{b} \frac{(s-a)^{\alpha}}{\Gamma(\alpha+1)} d H(s)\right.\right. \\
& \left.\left.+\sum_{i=1}^{n}\left|\mu_{i}\right| \frac{\left(\xi_{i}-a\right)^{\alpha+1}-\left(\eta_{i}-a\right)^{\alpha+1}}{\Gamma(\alpha+2)}+\frac{(b-a)^{\alpha}}{\Gamma(\alpha+1)}\right]\right\} L\|x-y\| \\
& +\left\{|k|(b-a)+\frac{(b-a)^{\gamma-1}}{|\Lambda|}\left[|\lambda||k| \int_{a}^{b}(s-a) d H(s)\right.\right. \\
& \left.\left.+\frac{1}{2}|k| \sum_{i=1}^{n}\left|\mu_{i}\right|\left(\left(\xi_{i}-a\right)^{2}-\left(\eta_{i}-a\right)^{2}\right)+|k|(b-a)\right]\right\}\|x-y\| \\
= & \left(L \Omega+\Omega_{1}\right)\|x-y\| .
\end{aligned}
$$

Hence $\|\mathcal{A} x-\mathcal{A} y\| \leq\left(L \Omega+\Omega_{1}\right)\|x-y\|$. Consequently, $\mathcal{A}$ is a contraction as, by assumption, $L \Omega+\Omega_{1}<1$. We deduce, by the Banach contraction mapping principle, that $\mathcal{A}$ has a fixed point, which means that the sequential Hilfer boundary value problem (1.3) has a unique solution. This completes the proof.

Example 2.1 Consider the sequential boundary value problems for Hilfer-type sequential fractional differential equation involving Riemann-Stieltjes integral multi-strip boundary conditions

$$
\left\{\begin{array}{l}
\left({ }^{H} D^{\frac{3}{2}, \frac{2}{3}}+\frac{1}{9} H D^{\frac{1}{2}, \frac{2}{3}}\right) x(t)=\frac{8 e^{-\left(\frac{1}{8}-t\right)^{2}}}{8 t+175}\left(\frac{x^{2}(t)+2|x(t)|}{1+|x(t)|}\right)+\frac{1}{2}, \quad t \in\left[\frac{1}{8}, \frac{15}{8}\right] \\
x\left(\frac{1}{8}\right)=0 \\
x\left(\frac{15}{8}\right)=\frac{1}{16} \int_{\frac{1}{8}}^{\frac{15}{8}} x(s) d\left(e^{-s} s^{\frac{1}{2}}\right) \\
\quad+\frac{3}{13} \int_{\frac{1}{4}}^{\frac{5}{8}} x(s) d s+\frac{5}{14} \int_{\frac{3}{4}}^{\frac{5}{4}} x(s) d s+\frac{7}{15} \int_{\frac{11}{8}}^{\frac{7}{4}} x(s) d s .
\end{array}\right.
$$


The above problem is a special case of problem (1.3), by taking $a=1 / 8, b=15 / 8$, $\alpha=3 / 2, \beta=2 / 3, k=1 / 9, \lambda=1 / 16, H(s)=e^{-s} s^{\frac{1}{2}}, n=3, \mu_{1}=3 / 13, \mu_{2}=5 / 14, \mu_{3}=7 / 15$, $\eta_{1}=1 / 4, \eta_{2}=3 / 4, \eta_{3}=11 / 8, \xi_{1}=5 / 8, \xi_{2}=5 / 4, \xi_{3}=7 / 4, \gamma=3 / 2+(2-(3 / 2))(2 / 3)=11 / 6$. From these constants, we can compute that $\Lambda \approx 1.179406838, \Omega \approx 4.551972757$, and $\Omega_{1} \approx 0.5203741066$.

Since

$$
f(t, x)=\frac{8 e^{-\left(\frac{1}{8}-t\right)^{2}}}{8 t+175}\left(\frac{x^{2}+2|x|}{1+|x|}\right)+\frac{1}{2}
$$

we get the inequality $|f(t, x)-f(t, y)| \leq(1 / 11)|x-y|$ satisfying condition $\left(H_{1}\right)$ of Theorem 2.1 with $L=1 / 11$. On the other hand, obviously we have

$$
L \Omega+\Omega_{1} \approx 0.9341898118<1 .
$$

Hence all the conditions of Theorem 2.1 are satisfied. In consequence, by Theorem 2.1, problem (2.10) has a unique solution on $[1 / 8,15 / 8]$.

\subsection{Existence results}

Two existence results are presented in this subsection. The first one is based on the wellknown Krasnosel'skii fixed point theorem [28].

Theorem 2.2 Let $f:[a, b] \times \mathbb{R} \rightarrow \mathbb{R}$ be a continuous function such that:

$\left(H_{2}\right)|f(t, w)| \leq \varphi(t), \forall(t, w) \in[a, b] \times \mathbb{R}$, and $\varphi \in C\left([a, b], \mathbb{R}^{+}\right)$.

Then, if $\Omega_{1}<1$, where $\Omega_{1}$ is defined in (2.7), the sequential Hilfer boundary value problem (1.3) has on $[a, b]$ at least one solution.

Proof We set $\sup _{t \in[a, b]} \varphi(t)=\|\varphi\|$, choose

$$
\rho \geq \frac{\|\varphi\| \Omega}{1-\Omega_{1}}
$$

and consider $B_{\rho}=\{x \in C([a, b], \mathbb{R}):\|x\| \leq \rho\}$. We define the operators $\mathcal{A}_{1}, \mathcal{A}_{2}$ on $B_{\rho}$ by

$$
\begin{aligned}
\mathcal{A}_{1} x(t)= & I^{\alpha} f(t, x(t))+\frac{(t-a)^{\gamma-1}}{\Lambda}\left[\lambda \int_{a}^{b} I^{\alpha} f(s, x(s)) d H(s)\right. \\
& \left.+\sum_{i=1}^{n} \mu_{i} \int_{\eta_{i}}^{\xi_{i}} I^{\alpha} f(s, x(s)) d s-I^{\alpha} f(b, x(b))\right], \quad t \in[a, b],
\end{aligned}
$$

and

$$
\begin{aligned}
\mathcal{A}_{2} x(t)= & -k \int_{a}^{t} x(s) d s+\frac{(t-a)^{\gamma-1}}{\Lambda}\left[-\lambda k \int_{a}^{b} \int_{a}^{s} x(u) d u d H(s)\right. \\
& \left.-k \sum_{i=1}^{n} \mu_{i} \int_{\eta_{i}}^{\xi_{i}} \int_{a}^{s} x(u) d u d s+k \int_{a}^{b} x(s) d s\right], \quad t \in[a, b] .
\end{aligned}
$$


For $x, y \in B_{\rho}$, we have

$$
\begin{aligned}
\left|\left(\mathcal{A}_{1} x\right)(t)+\left(\mathcal{A}_{2} y\right)(t)\right| & I^{\alpha}|f(t, x(t))|+|k| \int_{a}^{t}|y(s)| d s \\
& +\frac{(b-a)^{\gamma-1}}{|\Lambda|}\left[|\lambda| \int_{a}^{b}\left[|k| \int_{a}^{s}|y(u)| d u+I^{\alpha}|f(s, x(s))|\right] d H(s)\right. \\
& +|k| \sum_{i=1}^{n}\left|\mu_{i}\right| \int_{\eta_{i}}^{\xi_{i}} \int_{a}^{s}|y(u)| d u d s+\sum_{i=1}^{n}\left|\mu_{i}\right| \int_{\eta_{i}}^{\xi_{i}} I^{\alpha}|f(s, x(s))| d s \\
& \left.+|k| \int_{a}^{b}|y(s)| d s+I^{\alpha}|f(b, x(b))|\right] \\
\leq & \left\{\frac{(b-a)^{\alpha}}{\Gamma(\alpha+1)}+\frac{(b-a)^{\gamma-1}}{|\Lambda|}\left[|\lambda| \int_{a}^{b} \frac{(s-a)^{\alpha}}{\Gamma(\alpha+1)} d H(s)\right.\right. \\
\leq & +\varphi \| \Omega+\Omega_{1} \rho \leq \rho . \\
& \left.\left.+\sum_{i=1}^{n}\left|\mu_{i}\right| \frac{\left(\xi_{i}-a\right)^{\alpha+1}-\left(\eta_{i}-a\right)^{\alpha+1}}{\Gamma(\alpha+2)}+\frac{(b-a)^{\alpha}}{\Gamma(\alpha+1)}\right]\right\}\|\varphi\| \\
& +\left\{|k|(b-a)+\frac{(b-a)^{\gamma-1}}{|\Lambda|}\left[|\lambda||k| \int_{a}^{b}(s-a) d H(s)\right.\right. \\
& \left\{\begin{array}{l}
n \\
2
\end{array}\left|\mu_{i}\right|\left(\left(\xi_{i}-a\right)^{2}-\left(\eta_{i}-a\right)^{2}\right)+|k|(b-a)\right]\|x\|
\end{aligned}
$$

Therefore $\left\|\mathcal{A}_{1} x+\mathcal{A}_{2} y\right\| \leq \rho$, which shows that $\mathcal{A}_{1} x+\mathcal{A}_{2} y \in B_{\rho}$. It is easy to see, using the condition $\Omega_{1}<1$, that $\mathcal{A}_{2}$ is a contraction mapping.

The operator $\mathcal{A}_{1}$ is continuous since $f$ is continuous. Moreover, the uniform boundedness on $B_{\rho}$ of the operator $\mathcal{A}_{1}$ follows from the relation

$$
\begin{aligned}
\left\|\mathcal{A}_{1} x\right\| \leq & \left\{\frac{(b-a)^{\alpha}}{\Gamma(\alpha+1)}+\frac{(b-a)^{\gamma-1}}{|\Lambda|}\left[|\lambda| \int_{a}^{b} \frac{(s-a)^{\alpha}}{\Gamma(\alpha+1)} d H(s)\right.\right. \\
& \left.\left.+\sum_{i=1}^{n}\left|\mu_{i}\right| \frac{\left(\xi_{i}-a\right)^{\alpha+1}-\left(\eta_{i}-a\right)^{\alpha+1}}{\Gamma(\alpha+2)}+\frac{(b-a)^{\alpha}}{\Gamma(\alpha+1)}\right]\right\}\|\varphi\| .
\end{aligned}
$$

Now we prove the equicontinuity of the operator $\mathcal{A}_{1}$. Let $t_{1}, t_{2} \in[a, b]$ with $t_{1}<t_{2}$. Then we have

$$
\begin{aligned}
& \left|\left(\mathcal{A}_{1} x\right)\left(t_{2}\right)-\left(\mathcal{A}_{1} x\right)\left(t_{1}\right)\right| \\
& \leq \leq \frac{1}{\Gamma(\alpha)}\left|\int_{a}^{t_{1}}\left[\left(t_{2}-s\right)^{\alpha-1}-\left(t_{1}-s\right)^{\alpha-1}\right] f(s, x(s)) d s+\int_{t_{1}}^{t_{2}}\left(t_{2}-s\right)^{\alpha-1} f(s, x(s)) d s\right| \\
& \quad+\frac{\left(t_{2}-a\right)^{\gamma-1}-\left(t_{1}-a\right)^{\gamma-1}}{|\Lambda|}\left[|\lambda| \int_{a}^{b} I^{\alpha}|f(s, x(s))| d H(s)\right.
\end{aligned}
$$




$$
\begin{aligned}
& +\sum_{i=1}^{n}\left|\mu_{i}\right| \int_{\eta_{i}}^{\xi_{i}} I^{\alpha}|f(s, x(s))| d s+I^{\alpha} \mid f(b, x(b) \mid] \\
\leq & \frac{\|\phi\|}{\Gamma(\alpha+1)}\left[2\left(t_{2}-t_{1}\right)^{\alpha}+\left|\left(t_{2}-a\right)^{\alpha}-\left(t_{1}-a\right)^{\alpha}\right|\right] \\
& +\frac{\left(t_{2}-a\right)^{\gamma-1}-\left(t_{1}-a\right)^{\gamma-1}}{|\Lambda|}\|\phi\|\left[|\lambda| \int_{a}^{b} \frac{(s-a)^{\alpha}}{\Gamma(\alpha+1)} d H(s)\right. \\
& \left.+\sum_{i=1}^{n}\left|\mu_{i}\right| \frac{\left(\xi_{i}-a\right)^{\alpha+1}-\left(\eta_{i}-a\right)^{\alpha+1}}{\Gamma(\alpha+2)}+\frac{(b-a)^{\alpha}}{\Gamma(\alpha+1)}\right] \\
\rightarrow & 0,
\end{aligned}
$$

as $t_{2}-t_{1} \rightarrow 0$ and is independent of $x$. Therefore, the operator $\mathcal{A}_{1}$ is equicontinuous. By the Arzelá-Ascoli theorem, $\mathcal{A}_{1}$ is compact on $B_{\rho}$. Consequently all the assumptions of Krasnosel'skiii's fixed point theorem [28] are satisfied. Hence the sequential Hilfer boundary value problem (1.3) has at least one solution on $[a, b]$. The proof is completed.

In our second existence result we apply the Leray-Schauder nonlinear alternative [29].

Theorem 2.3 Let $\Omega_{1}<1$. In addition, we assume that:

$\left(H_{3}\right)|f(t, w)| \leq p(t) \psi(|w|)$ for each $(t, w) \in[a, b] \times \mathbb{R}$, where $\psi:[0, \infty) \rightarrow(0, \infty)$ is a nondecreasing and continuous function and $p \in C\left([a, b], \mathbb{R}^{+}\right)$;

$\left(H_{4}\right) \frac{\left(1-\Omega_{1}\right) K}{\psi(K)\|p\| \Omega}>1$ for a constant $K>0$.

Then the sequential Hilfer boundary value problem (1.3) has at least one solution on $[a, b]$.

Proof Consider the operator $\mathcal{A}$ defined by (2.5). We shall show that bounded sets are mapped by $\mathcal{A}$ into a bounded set in $C([a, b], \mathbb{R})$. For $r>0$, let $B_{r}=\{x \in C([a, b], \mathbb{R}):\|x\| \leq$ $r\}$. For any $t \in[a, b]$, we have

$$
\begin{aligned}
|(\mathcal{A} x)(t)| & \\
\leq & I^{\alpha}|f(t, x(t))|+|k| \int_{a}^{t}|x(s)| d s \\
& +\frac{(b-a)^{\gamma-1}}{|\Lambda|}\left[|\lambda| \int_{a}^{b}\left[|k| \int_{a}^{s}|x(u)| d u+I^{\alpha}|f(s, x(s))|\right] d H(s)\right. \\
& +|k| \sum_{i=1}^{n}\left|\mu_{i}\right| \int_{\eta_{i}}^{\xi_{i}} \int_{a}^{s}|x(u)| d u d s+\sum_{i=1}^{n}\left|\mu_{i}\right| \int_{\eta_{i}}^{\xi_{i}} I^{\alpha}|f(s, x(s))| d s \\
& \left.+|k| \int_{a}^{b}|x(s)| d s+I^{\alpha}|f(b, x(b))|\right] \\
\leq & \left\{\frac{(b-a)^{\alpha}}{\Gamma(\alpha+1)}+\frac{(b-a)^{\gamma-1}}{|\Lambda|}\left[|\lambda| \int_{a}^{b} \frac{(s-a)^{\alpha}}{\Gamma(\alpha+1)} d H(s)\right.\right. \\
& \left.\left.+\sum_{i=1}^{n}\left|\mu_{i}\right| \frac{\left(\xi_{i}-a\right)^{\alpha+1}-\left(\eta_{i}-a\right)^{\alpha+1}}{\Gamma(\alpha+2)}+\frac{(b-a)^{\alpha}}{\Gamma(\alpha+1)}\right]\right\}\|p\| \psi(\|x\|) \\
& +\left\{|k|(b-a)+\frac{(b-a)^{\gamma-1}}{|\Lambda|}\left[|\lambda||k| \int_{a}^{b}(s-a) d H(s)\right.\right.
\end{aligned}
$$




$$
\begin{aligned}
& \left.\left.\quad+\frac{1}{2}|k| \sum_{i=1}^{n}\left|\mu_{i}\right|\left(\left(\xi_{i}-a\right)^{2}-\left(\eta_{i}-a\right)^{2}\right)+|k|(b-a)\right]\right\}\|x\| \\
& \leq\|p\| \psi(\|x\|) \Omega+\Omega_{1}\|x\| \leq r,
\end{aligned}
$$

and consequently,

$$
\|\mathcal{A} x\| \leq\|p\| \psi(r)\|p\| \Omega+\Omega_{1} r
$$

In the next step we will show that bounded sets are mapped by the operator $\mathcal{A}$ into equicontinuous sets of $C([a, b], \mathbb{R})$. Let $t_{1}, t_{2} \in[a, b]$ with $t_{1}<t_{2}$ and $x \in B_{r}$. Then we have

$$
\begin{aligned}
&\left|(\mathcal{A} x)\left(t_{2}\right)-(\mathcal{A} x)\left(t_{1}\right)\right| \\
& \leq \frac{1}{\Gamma(\alpha)} \mid \int_{a}^{t_{1}}\left[\left(t_{2}-s\right)^{\alpha-1}-\left(t_{1}-s\right)^{\alpha-1}\right] f(s, x(s)) d s \\
&+\int_{t_{1}}^{t_{2}}\left(t_{2}-s\right)^{\alpha-1} f(s, x(s)) d s|+| k \mid r\left(t_{2}-t_{1}\right) \\
&+\frac{\left(t_{2}-a\right)^{\gamma-1}-\left(t_{1}-a\right)^{\gamma-1}}{|\Lambda|}\|p\| \psi(r)\left[|\lambda| \int_{a}^{b} \frac{(s-a)^{\alpha}}{\Gamma(\alpha+1)} d H(s)\right. \\
&\left.+\sum_{i=1}^{n}\left|\mu_{i}\right| \frac{\left(\xi_{i}-a\right)^{\alpha+1}-\left(\eta_{i}-a\right)^{\alpha+1}}{\Gamma(\alpha+2)}+\frac{(b-a)^{\alpha}}{\Gamma(\alpha+1)}\right] \\
&+\frac{\left(t_{2}-a\right)^{\gamma-1}-\left(t_{1}-a\right)^{\gamma-1}}{|\Lambda|} r\left[|\lambda||k| \int_{a}^{b}(s-a) d H(s)\right. \\
&\left.+\frac{1}{2}|k| \sum_{i=1}^{n}\left|\mu_{i}\right|\left(\left(\xi_{i}-a\right)^{2}-\left(\eta_{i}-a\right)^{2}\right)+|k|(b-a)\right] \\
& \leq \frac{\|p\| \psi(r)}{\Gamma(\alpha+1)}\left[2\left(t_{2}-t_{1}\right)^{\alpha}+\left|\left(t_{2}-a\right)^{\alpha}-\left(t_{1}-a\right)^{\alpha}\right|\right]+|k| r\left(t_{2}-t_{1}\right) \\
&+\frac{\left(t_{2}-a\right)^{\gamma-1}-\left(t_{1}-a\right)^{\gamma-1}}{|\Lambda|}\|p\| \psi(r)\left[|\lambda| \int_{a}^{b} \frac{(s-a)^{\alpha}}{\Gamma(\alpha+1)} d H(s)\right. \\
&\left.+\frac{1}{2}|k| \sum_{i=1}^{n}\left|\mu_{i}\right|\left(\left(\xi_{i}-a\right)^{2}-\left(\eta_{i}-a\right)^{2}\right)+|k|(b-a)\right] \\
&\left.+\sum_{i=1}^{n}\left|\mu_{i}\right| \frac{\left(\xi_{i}-a\right)^{\alpha+1}-\left(\eta_{i}-a\right)^{\alpha+1}}{\Gamma(\alpha+2)}+\frac{(b-a)^{\alpha}}{\Gamma(\alpha+1)}\right] \\
& \frac{\left(t_{2}-a\right)^{\gamma-1}-\left(t_{1}-a\right)^{\gamma-1}}{|\Lambda|}\left[|\lambda||k| \int_{a}^{b}(s-a) d H(s)\right. \\
&
\end{aligned}
$$

as $t_{2}-t_{1} \rightarrow 0$ and is independent of $x \in B_{r}$. By the Arzelá-Ascoli theorem, the operator $\mathcal{A}: C([a, b], \mathbb{R}) \rightarrow C([a, b], \mathbb{R})$ is completely continuous.

Finally we will prove that the set of all solutions to equation $x=\theta \mathcal{A} x$ for $\theta \in(0,1)$ is bounded. 
Let $x$ be a solution of the sequential Hilfer boundary value problem (1.3). Then, as in the first step, for $t \in[a, b]$, we have

$$
|x(t)| \leq \psi(\|x\|)\|p\| \Omega+\Omega_{1}\|x\|
$$

or

$$
\frac{\left(1-\Omega_{1}\right)\|x\|}{\psi(\|x\|)\|p\| \Omega} \leq 1
$$

By $\left(H_{4}\right),\|x\| \neq K$ for some $K$. We set

$$
U=\{x \in C([a, b], \mathbb{R}):\|x\|<K\} .
$$

The operator $\mathcal{A}: \bar{U} \rightarrow C([a, b], \mathbb{R})$ is continuous and completely continuous. There is no $x \in \partial U$ such that $x=\theta \mathcal{A} x$ for some $\theta \in(0,1)$, from the choice of $U$. By the nonlinear alternative of Leray-Schauder type [29], we conclude that the operator $\mathcal{A}$ has a fixed point $x \in \bar{U}$. Obviously this fixed point is a solution of the sequential Hilfer boundary value problem (1.3). This finishes the proof.

Example 2.2 Consider the sequential boundary value problems for Hilfer-type sequential fractional differential equation involving Riemann-Stieltjes integral multi-strip boundary conditions

$$
\left\{\begin{array}{l}
\left({ }^{H} D^{\frac{7}{4}, \frac{1}{2}}+\frac{1}{17} D^{H} D^{\frac{3}{4}, \frac{1}{2}}\right) x(t)=f(t, x(t)), \quad t \in\left[\frac{1}{9}, \frac{14}{9}\right], \\
x\left(\frac{1}{9}\right)=0 \\
x\left(\frac{14}{9}\right)=\frac{2}{11} \int_{\frac{1}{9}}^{\frac{14}{9}} x(s) d\left(s^{-\frac{1}{3}} \sin s\right)+\frac{4}{21} \int_{\frac{2}{9}}^{\frac{4}{9}} x(s) d s \\
\quad+\frac{6}{31} \int_{\frac{5}{9}}^{\frac{7}{9}} x(s) d s+\frac{8}{41} \int_{\frac{8}{9}}^{\frac{10}{9}} x(s) d s+\frac{10}{51} \int_{\frac{11}{9}}^{\frac{13}{9}} x(s) d s .
\end{array}\right.
$$

By putting, $a=1 / 9, b=14 / 9, \alpha=7 / 4, \beta=1 / 2, k=1 / 17, \lambda=2 / 11, H(s)=s^{-\frac{1}{3}} \sin s, n=4$, $\mu_{1}=4 / 21, \mu_{2}=6 / 31, \mu_{3}=8 / 41, \mu_{4}=10 / 51, \eta_{1}=2 / 9, \eta_{2}=5 / 9, \eta_{3}=8 / 9, \eta_{4}=11 / 9, \xi_{1}=4 / 9$, $\xi_{2}=7 / 9, \xi_{3}=10 / 9, \xi_{4}=13 / 9, \gamma=7 / 4+(2-(7 / 4))(1 / 2)=15 / 8$, we verify the conditions that $\Lambda \approx 1.206968182, \Omega \approx 2.635069870$, and $\Omega_{1} \approx 0.1932006417$.

(i) If the nonlinear function $f(t, x)$ in $(2.11)$ is given by

$$
f(t, x)=t^{2} e^{-x^{2}}+2 t \frac{x^{8}}{1+x^{8}} \cos ^{2} x^{4}+\frac{1}{3},
$$

then $f$ is continuous on $[1 / 9,14 / 9] \times \mathbb{R}$ and $|f(t, x)| \leq t^{2}+2 t+(1 / 3)$ satisfying condition $\left(H_{2}\right)$ in Theorem 2.2. Since $\Omega_{1}<1$, we conclude by Theorem 2.2 that problem (2.11) with (2.12) has at least one solution on $[1 / 9,14 / 9]$.

(ii) Let the nonlinear function $f(t, x)$ in $(2.11)$ be defined by

$$
f(t, x)=\frac{9}{9 t+89}\left(\frac{x^{12}}{1+x^{10}}+\frac{x^{16}}{4\left(1+x^{16}\right)} e^{-x^{2}}+\frac{3}{4}\right) .
$$

Thus $f$ fulfills condition $\left(H_{3}\right)$ in Theorem 2.3 that $|f(t, x)| \leq(9 /(9 t+89))\left(x^{2}+1\right)$ with $p(t)=9 /(9 t+89)$ and $\psi(x)=x^{2}+1$. Since $\|p\|=1 / 10$, there exists a constant $K \in$ $(0.371742602,2.690033358)$ satisfying inequality in $\left(H_{4}\right)$. In consequence of Theorem 2.3, 
the sequential boundary value problem (2.11) with (2.13) has at least one solution on $[1 / 9,14 / 9]$.

\section{Existence results for problem (1.4)}

In the following we use the notation $\mathcal{P}_{c p, c}(X)=\{Y \in \mathcal{P}(X): Y$ is compact and convex $\}$ for a normed space $(X,\|\cdot\|)$. For details on multi-valued analysis, we refer to the monographs [7, 30-32].

Definition 3.1 A function $x \in C^{2}([a, b], \mathbb{R})$ is a solution of the sequential Hilfer boundary value problem (1.4) if $x(a)=0, x(b)=\lambda \int_{a}^{b} x(s) d H(s)+\sum_{i=1}^{n} \mu_{i} \int_{\eta_{i}}^{\xi_{i}} x(s) d s$, and there exists a function $v \in L^{1}([a, b], \mathbb{R})$ such that $v(t) \in F(t, x(t))$ a.e. on $[a, b]$ and

$x(t)$

$$
\begin{aligned}
= & I^{\alpha} v(t)-k \int_{a}^{t} x(s) d s+\frac{(t-a)^{\gamma-1}}{\Lambda}\left[-\lambda \int_{a}^{b}\left[k \int_{a}^{s} x(u) d u-I^{\alpha} v(s)\right] d H(s)\right. \\
& \left.-k \sum_{i=1}^{n} \mu_{i} \int_{\eta_{i}}^{\xi_{i}} \int_{a}^{s} x(u) d u d s+\sum_{i=1}^{n} \mu_{i} \int_{\eta_{i}}^{\xi_{i}} I^{\alpha} v(s) d s+k \int_{a}^{b} x(s) d s-I^{\alpha} v(b)\right] .
\end{aligned}
$$

Theorem 3.1 Assume that $\Omega_{1}<1$. In addition, we suppose that:

$\left(A_{1}\right) F:[a, b] \times \mathbb{R} \rightarrow \mathcal{P}_{c p, c}(\mathbb{R})$ is an $L^{1}$-Carathéodory multi-valued map, i.e., (i) $t \longmapsto$ $F(t, x)$ is measurable for each $x \in \mathbb{R}$; (ii) $x \mapsto F(t, x)$ is upper semicontinuous for almost all $t \in[a, b]$; (iii) for each $\alpha>0$, there exists $\varphi_{\alpha} \in L^{1}\left([a, b], \mathbb{R}^{+}\right)$such that

$$
\|F(t, x)\|=\sup \{|v|: v \in F(t, x)\} \leq \varphi_{\alpha}(t)
$$

for all $x \in \mathbb{R}$ with $\|x\| \leq \alpha$ and for a.e. $t \in[a, b] ;$

$\left(A_{2}\right)\|F(t, z)\|_{\mathcal{P}}:=\sup \{|y|: y \in F(t, z)\} \leq p(t) \psi(\|z\|)$ for each $(t, z) \in[a, b] \times \mathbb{R}$, where $\psi$ : $[0, \infty) \rightarrow(0, \infty)$ is a nondecreasing continuous function and $p \in C\left([a, b], \mathbb{R}^{+}\right)$;

$\left(A_{3}\right)$ There exists a constant $M>0$ such that

$$
\frac{M}{\|p\| \psi(M) \Omega}>\frac{1}{1-\Omega_{1}}
$$

where $\Omega$ and $\Omega_{1}$ are given in (2.6) and (2.7) respectively.

Then the sequential Hilfer boundary value problem (1.4) has at least one solution on $[a, b]$.

Proof We transform the sequential Hilfer boundary value problem (1.4) into a fixed point problem. To do this, we define an operator $\mathcal{N}: C([a, b], \mathbb{R}) \longrightarrow \mathcal{P}(C([a, b], \mathbb{R}))$ by

$$
\mathcal{N}(x)=\left\{\begin{array}{l}
h \in C([a, b], \mathbb{R}): \\
h(t)=\left\{\begin{array}{l}
I^{\alpha} v(t)-k \int_{a}^{t} x(s) d s \\
+\frac{(t-a)^{\gamma-1}}{\Lambda}\left[-\lambda \int_{a}^{b}\left[k \int_{a}^{s} x(u) d u-I^{\alpha} v(s)\right] d H(s)\right. \\
-k \sum_{i=1}^{n} \mu_{i} \int_{\eta_{i}}^{\xi_{i}} \int_{a}^{s} x(u) d u d s+\sum_{i=1}^{n} \mu_{i} \int_{\eta_{i}}^{\xi_{i}} I^{\alpha} v(s) d s \\
\left.+k \int_{a}^{b} x(s) d s-I^{\alpha} v(b)\right]
\end{array}\right.
\end{array}\right.
$$

for $v \in S_{F, x}$. 
Next we introduce the single-valued operator $\mathcal{A}: C([a, b], \mathbb{R}) \longrightarrow C([a, b], \mathbb{R})$ and the multi-valued operator $\mathcal{B}: C([a, b], \mathbb{R}) \longrightarrow \mathcal{P}(C([a, b], \mathbb{R}))$ by

$$
\begin{aligned}
\mathcal{A} x(t)= & -k \int_{a}^{t} x(s) d s+\frac{(t-a)^{\gamma-1}}{\Lambda}\left[-\lambda k \int_{a}^{b} \int_{a}^{s} x(u) d u d H(s)\right. \\
& \left.-k \sum_{i=1}^{n} \mu_{i} \int_{\eta_{i}}^{\xi_{i}} \int_{a}^{s} x(u) d u d s+k \int_{a}^{b} x(s) d s\right],
\end{aligned}
$$

and

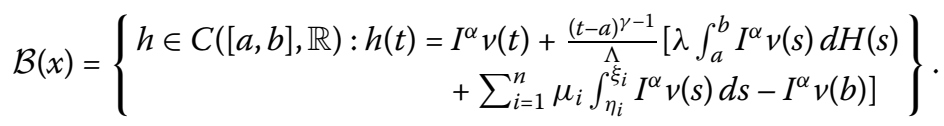

Observe that $\mathcal{N}=\mathcal{A}+\mathcal{B}$. To prove our existence result, we make use of the nonlinear alternative for contractive maps [33, Corollary 3.8].

Step 1: First we show that $\mathcal{A}$ is a contraction on $C([a, b], \mathbb{R})$. We have, for $x, y \in$ $C([a, b], \mathbb{R})$,

$$
\begin{aligned}
|\mathcal{A} x(t)-\mathcal{A} y(t)| \leq & \left\{|k|(b-a)+\frac{(b-a)^{\gamma-1}}{|\Lambda|}\left[|\lambda||k| \int_{a}^{b}(s-a) d H(s)\right.\right. \\
& \left.\left.+\frac{1}{2}|k| \sum_{i=1}^{n}\left|\mu_{i}\right|\left(\left(\xi_{i}-a\right)^{2}-\left(\eta_{i}-a\right)^{2}\right)+|k|(b-a)\right]\right\}\|x-y\| \\
\leq & \Omega_{1}\|x-y\|,
\end{aligned}
$$

which yields

$$
\|\mathcal{A} x-\mathcal{A} y\| \leq \Omega_{1}\|x-y\| .
$$

Hence, since $\Omega_{1}<1$, the operator $\mathcal{A}$ is a contraction.

Step 2: For all $x \in C([a, b], \mathbb{R}), \mathcal{B}(x)$ is convex. Let $z_{1}, z_{2} \in \mathcal{B}(x)$. Then there exist $v_{1}, v_{2} \in$ $S_{F, x}$ such that

$$
\begin{aligned}
& z_{i}(t)=I^{\alpha} v_{i}(t)+\frac{(t-a)^{\gamma-1}}{\Lambda}\left[\lambda \int_{a}^{b} I^{\alpha} v_{i}(s) d H(s)+\sum_{i=1}^{n} \mu_{i} \int_{\eta_{i}}^{\xi_{i}} I^{\alpha} v_{i}(s) d s-I^{\alpha} v_{i}(b)\right], \\
& \quad i=1,2,
\end{aligned}
$$

for almost all $t \in[a, b]$. Let $0 \leq \theta \leq 1$. Then we have

$$
\begin{aligned}
{\left[\theta z_{1}+(1-\theta) z_{2}\right](t)=} & I^{\alpha}\left[\theta v_{1}+(1-\theta) v_{2}\right](t) \\
& +\frac{(t-a)^{\gamma-1}}{\Lambda}\left[\lambda \int_{a}^{b} I^{\alpha}\left[\theta v_{1}+(1-\theta) v_{2}\right](s) d H(s)\right. \\
& \left.+\sum_{i=1}^{n} \mu_{i} \int_{\eta_{i}}^{\xi_{i}} I^{\alpha}\left[\theta v_{1}+(1-\theta) v_{2}\right](s) d s-I^{\alpha}\left[\theta v_{1}+(1-\theta) v_{2}\right](b)\right] .
\end{aligned}
$$


The fact that $F$ has convex values implies that $S_{F, x}$ is convex, and thus $\theta v_{1}(s)+(1-$ $\theta) v_{2}(s) \in S_{F, x}$. Hence $\theta z_{1}+(1-\theta) z_{2} \in \mathcal{B}(x)$, which proves that the operator $\mathcal{B}$ is convexvalued.

Step 3: Now we will prove that the operator $\mathcal{B}$ is compact and upper semicontinuous.

First, we prove that bounded sets are mapped by $\mathcal{B}$ into bounded sets in $C([a, b]$, $\mathbb{R})$. Let $B_{r}=\{x \in C([a, b], \mathbb{R}):\|x\| \leq r\}$. For each $h \in \mathcal{B}(x), x \in B_{r}$, there exists $v \in S_{F, x}$ such that

$$
\begin{aligned}
& h(t)=I^{\alpha} v(t)+\frac{(t-a)^{\gamma-1}}{\Lambda}\left[\lambda \int_{a}^{b} I^{\alpha} v(s) d H(s)+\sum_{i=1}^{n} \mu_{i} \int_{\eta_{i}}^{\xi_{i}} I^{\alpha} v(s) d s-I^{\alpha} v(b)\right] \\
& t \in[a, b]
\end{aligned}
$$

We have, for $t \in[a, b]$,

$$
\begin{aligned}
|h(t)| \leq & I^{\alpha}|\nu(t)|+\frac{(b-a)^{\gamma-1}}{|\Lambda|}\left[|\lambda| \int_{a}^{b} I^{\alpha}|\nu(s)| d H(s)\right. \\
& \left.+\sum_{i=1}^{n}\left|\mu_{i}\right| \int_{\eta_{i}}^{\xi_{i}} I^{\alpha}|\nu(s)| d s+I^{\alpha}|\nu(b)|\right] \\
\leq & \|p\| \psi(\|x\|)\left\{\frac{(b-a)^{\alpha}}{\Gamma(\alpha+1)}+\frac{(b-a)^{\gamma-1}}{|\Lambda|}\left[|\lambda| \int_{a}^{b} \frac{(s-a)^{\alpha}}{\Gamma(\alpha+1)} d H(s)\right.\right. \\
& \left.\left.+\sum_{i=1}^{n}\left|\mu_{i}\right| \frac{\left(\xi_{i}-a\right)^{\alpha+1}-\left(\eta_{i}-a\right)^{\alpha+1}}{\Gamma(\alpha+2)}+\frac{(b-a)^{\alpha}}{\Gamma(\alpha+1)}\right]\right\} .
\end{aligned}
$$

Thus,

$$
\begin{aligned}
\|h\| \leq & \|p\| \psi(r)\left\{\frac{(b-a)^{\alpha}}{\Gamma(\alpha+1)}+\frac{(b-a)^{\gamma-1}}{|\Lambda|}\left[|\lambda| \int_{a}^{b} \frac{(s-a)^{\alpha}}{\Gamma(\alpha+1)} d H(s)\right.\right. \\
& \left.\left.+\sum_{i=1}^{n}\left|\mu_{i}\right| \frac{\left(\xi_{i}-a\right)^{\alpha+1}-\left(\eta_{i}-a\right)^{\alpha+1}}{\Gamma(\alpha+2)}+\frac{(b-a)^{\alpha}}{\Gamma(\alpha+1)}\right]\right\} .
\end{aligned}
$$

Secondly we prove that bounded sets are mapped by the operator $\mathcal{B}$ into equicontinuous sets. Let $t_{1}, t_{2} \in[a, b]$ with $t_{1}<t_{2}$ and $x \in B_{r}$. Then, for each $h \in \mathcal{B}(x)$, we obtain

$$
\begin{aligned}
\left|h\left(t_{2}\right)-h\left(t_{1}\right)\right|= & \frac{1}{\Gamma(\alpha)} \mid \int_{a}^{t_{1}}\left[\left(t_{2}-s\right)^{\alpha-1}-\left(t_{1}-s\right)^{\alpha-1}\right] v(s) d s \\
& +\int_{t_{1}}^{t_{2}}\left(t_{2}-s\right)^{\alpha-1} v(s) d s|+| k \mid r\left(t_{2}-t_{1}\right) \\
& +\frac{\left(t_{2}-a\right)^{\gamma-1}-\left(t_{1}-a\right)^{\gamma-1}}{|\Lambda|}\|p\| \psi(r)\left[|\lambda| \int_{a}^{b} \frac{(s-a)^{\alpha}}{\Gamma(\alpha+1)} d H(s)\right. \\
& \left.+\sum_{i=1}^{n}\left|\mu_{i}\right| \frac{\left(\xi_{i}-a\right)^{\alpha+1}-\left(\eta_{i}-a\right)^{\alpha+1}}{\Gamma(\alpha+2)}+\frac{(b-a)^{\alpha}}{\Gamma(\alpha+1)}\right] \\
\leq & \frac{\|p\| \psi(r)}{\Gamma(\alpha+1)}\left[2\left(t_{2}-t_{1}\right)^{\alpha}+\left|\left(t_{2}-a\right)^{\alpha}-\left(t_{1}-a\right)^{\alpha}\right|\right]
\end{aligned}
$$




$$
\begin{aligned}
& +\frac{\left(t_{2}-a\right)^{\gamma-1}-\left(t_{1}-a\right)^{\gamma-1}}{|\Lambda|}\|p\| \psi(r)\left[|\lambda| \int_{a}^{b} \frac{(s-a)^{\alpha}}{\Gamma(\alpha+1)} d H(s)\right. \\
& \left.+\sum_{i=1}^{n}\left|\mu_{i}\right| \frac{\left(\xi_{i}-a\right)^{\alpha+1}-\left(\eta_{i}-a\right)^{\alpha+1}}{\Gamma(\alpha+2)}+\frac{(b-a)^{\alpha}}{\Gamma(\alpha+1)}\right] \\
& \rightarrow 0
\end{aligned}
$$

as $t_{2}-t_{1} \rightarrow 0$ and is independent of $x \in B_{r}$. By the Arzelá-Ascoli theorem the operator $\mathcal{B}: C([a, b], \mathbb{R}) \rightarrow \mathcal{P}(C([a, b], \mathbb{R}))$ is completely continuous.

By [30, Proposition 1.2] we know that a completely continuous operator is upper semicontinuous if it has a closed graph. Thus in the following we will prove that the operator $\mathcal{B}$ has a closed graph.

Let $x_{n} \rightarrow x_{*}, h_{n} \in \mathcal{B}\left(x_{n}\right)$ and $h_{n} \rightarrow h_{*}$. We shall show that $h_{*} \in \mathcal{B}\left(x_{*}\right)$. Since $h_{n} \in \mathcal{B}\left(x_{n}\right)$, there exists $v_{n} \in S_{F, x_{n}}$ such that, for each $t \in[a, b]$,

$$
h(t)=I^{\alpha} v_{n}(t)+\frac{(t-a)^{\gamma-1}}{\Lambda}\left[\lambda \int_{a}^{b} I^{\alpha} v_{n}(s) d H(s)+\sum_{i=1}^{n} \mu_{i} \int_{\eta_{i}}^{\xi_{i}} I^{\alpha} v_{n}(s) d s-I^{\alpha} v_{n}(b)\right] .
$$

It is suffices to show that there exists $v_{*} \in S_{F, x_{*}}$ such that, for each $t \in[a, b]$,

$$
h_{*}(t)=I^{\alpha} v_{*}(t)+\frac{(t-a)^{\gamma-1}}{\Lambda}\left[\lambda \int_{a}^{b} I^{\alpha} v_{*}(s) d H(s)+\sum_{i=1}^{n} \mu_{i} \int_{\eta_{i}}^{\xi_{i}} I^{\alpha} v_{*}(s) d s-I^{\alpha} v_{*}(b)\right] \text {. }
$$

Let us consider the linear operator $\Theta: L^{1}([a, b], \mathbb{R}) \rightarrow C([a, b], \mathbb{R})$ given by

$$
v \mapsto \Theta(v)(t)=I^{\alpha} v(t)+\frac{(t-a)^{\gamma-1}}{\Lambda}\left[\lambda \int_{a}^{b} I^{\alpha} v(s) d H(s)+\sum_{i=1}^{n} \mu_{i} \int_{\eta_{i}}^{\xi_{i}} I^{\alpha} v(s) d s-I^{\alpha} v(b)\right] .
$$

Observe that

$$
\begin{aligned}
\left\|h_{n}(t)-h_{*}(t)\right\|= & \| I^{\alpha}\left[v_{n}(t)-v_{*}(t)\right]+\frac{(t-a)^{\gamma-1}}{\Lambda}\left[|\lambda| \int_{a}^{b} I^{\alpha}\left[v_{n}(t)-v_{*}(t)\right] d H(s)\right. \\
& \left.+\sum_{i=1}^{n} \mu_{i} \int_{\eta_{i}}^{\xi_{i}} I^{\alpha}\left[v_{n}(t)-v_{*}(t)\right] d s-I^{\alpha}\left[v_{n}(b)-v_{*}(b)\right]\right] \| \\
\rightarrow & 0
\end{aligned}
$$

as $n \rightarrow \infty$.

Thus, it follows by a closed graph lemma [34] that $\Theta \circ S_{F}$ is a closed graph operator. Moreover, $h_{n}(t) \in \Theta\left(S_{F, x_{n}}\right)$. Since $x_{n} \rightarrow x_{*}$, we have that

$$
h_{*}(t)=I^{\alpha} v_{*}(t)+\frac{(t-a)^{\gamma-1}}{\Lambda}\left[\lambda \int_{a}^{b} I^{\alpha} v_{*}(s) d H(s)+\sum_{i=1}^{n} \mu_{i} \int_{\eta_{i}}^{\xi_{i}} I^{\alpha} v_{*}(s) d s-I^{\alpha} v_{*}(b)\right]
$$

for some $v_{*} \in S_{F, x_{*}}$. Thus the operator $\mathcal{B}$ has a closed graph.

Thus the operators $\mathcal{A}$ and $\mathcal{B}$ satisfy all the conditions of the nonlinear alternative for contractive maps [33, Corollary 3.8]. It implies that either (i) $\mathcal{N}$ has a fixed point in $[a, b]$ or (ii) there is a point $x \in \partial B_{M}=\{x \in C([a, b], \mathbb{R}):\|x\| \leq M\}$ and $\theta \in(0,1)$ with $x=\theta \mathcal{N}(x)$. 
In the next we will show that conclusion (ii) is not possible. If $x \in \theta \mathcal{A}(x)+\theta \mathcal{B}(x)$ for $\theta \in(0,1)$, then there exists $v \in S_{F, x}$ such that

$$
\begin{aligned}
& x(t) \\
& =I^{\alpha} v(t)-k \int_{a}^{t} x(s) d s+\frac{(t-a)^{\gamma-1}}{\Lambda}\left[-\lambda \int_{a}^{b}\left[k \int_{a}^{s} x(u) d u-I^{\alpha} v(s)\right] d H(s)\right. \\
& \left.\quad-k \sum_{i=1}^{n} \mu_{i} \int_{\eta_{i}}^{\xi_{i}} \int_{a}^{s} x(u) d u d s+\sum_{i=1}^{n} \mu_{i} \int_{\eta_{i}}^{\xi_{i}} I^{\alpha} v(s) d s+k \int_{a}^{b} x(s) d s-I^{\alpha} v(b)\right],
\end{aligned}
$$

and

$$
\begin{aligned}
|x(t)| \leq & \left\{\frac{(b-a)^{\alpha}}{\Gamma(\alpha+1)}+\frac{(b-a)^{\gamma-1}}{|\Lambda|}\left[|\lambda| \int_{a}^{b} \frac{(s-a)^{\alpha}}{\Gamma(\alpha+1)} d H(s)\right.\right. \\
& \left.\left.+\sum_{i=1}^{n}\left|\mu_{i}\right| \frac{\left(\xi_{i}-a\right)^{\alpha+1}-\left(\eta_{i}-a\right)^{\alpha+1}}{\Gamma(\alpha+2)}+\frac{(b-a)^{\alpha}}{\Gamma(\alpha+1)}\right]\right\}\|p\| \psi(\|x\|) \\
& +\left\{|k|(b-a)+\frac{(b-a)^{\gamma-1}}{|\Lambda|}\left[|\lambda||k| \int_{a}^{b}(s-a) d H(s)\right.\right. \\
& \left.\left.+\frac{1}{2}|k| \sum_{i=1}^{n}\left|\mu_{i}\right|\left(\left(\xi_{i}-a\right)^{2}-\left(\eta_{i}-a\right)^{2}\right)+|k|(b-a)\right]\right\}\|x\| \\
\leq & \|p\| \psi(\|x\|) \Omega+\Omega_{1}\|x\| .
\end{aligned}
$$

Thus

$$
\left(1-\Omega_{1}\right)\|x\| \leq\|p\| \psi(\|x\|) \Omega .
$$

If condition (ii) holds, then there exist $\theta \in(0,1)$ and $x \in \partial B_{M}$ with $x=\theta \mathcal{N}(x)$, which means that $x$ is a solution of (1.4) with $\|x\|=M$. By (3.5), we have

$$
\frac{M}{\|p\| \psi(M) \Omega} \leq \frac{1}{1-\Omega_{1}}
$$

which contradicts (3.2). Consequently, $\mathcal{N}$ has a fixed point in $[a, b]$. We deduce that the sequential Hilfer boundary value problem (1.4) has a solution. The proof is completed.

Example 3.1 Consider the boundary value problems for Hilfer-type sequential fractional differential inclusion involving Riemann-Stieltjes integral multi-strip boundary conditions

$$
\left\{\begin{array}{l}
\left({ }^{H} D^{\frac{4}{3}, \frac{2}{5}}+\frac{1}{12}{ }^{H} D^{\frac{1}{3}, \frac{2}{5}}\right) x(t) \in F(t, x(t)), \quad t \in\left[\frac{1}{7}, \frac{13}{7}\right], \\
x\left(\frac{1}{7}\right)=0 \\
x\left(\frac{13}{7}\right)=\frac{5}{44} \int_{\frac{1}{7}}^{\frac{13}{7}} x(s) d\left(s^{\frac{3}{2}}+2 s^{\frac{1}{2}}+s^{-\frac{1}{2}}\right) \\
\quad+\frac{6}{55} \int_{\frac{2}{7}}^{\frac{5}{7}} x(s) d s+\frac{7}{66} \int_{\frac{6}{7}}^{\frac{8}{7}} x(s) d s+\frac{8}{77} \int_{\frac{9}{7}}^{\frac{12}{7}} x(s) d s,
\end{array}\right.
$$


where

$$
F(t, x)=\left[\frac{7}{7 t+279}\left(\frac{x^{8}}{1+x^{6}}+\frac{1}{8} e^{-x^{4}}+\frac{1}{16}\right), \frac{7}{7 t+209}\left(\frac{x^{6}}{1+x^{4}}+\frac{1}{2} \cos ^{2} x+\frac{1}{2}\right)\right] .
$$

Here, $a=1 / 7, b=13 / 7, \alpha=4 / 3, \beta=2 / 5, k=1 / 12, \lambda=5 / 44, H(s)=s^{\frac{3}{2}}+2 s^{\frac{1}{2}}+s^{-\frac{1}{2}}, n=3$, $\mu_{1}=6 / 55, \mu_{2}=7 / 66, \mu_{3}=8 / 77, \eta_{1}=2 / 7, \eta_{2}=6 / 7, \eta_{3}=9 / 7, \xi_{1}=5 / 7, \xi_{2}=8 / 7, \xi_{3}=12 / 7$, $\gamma=4 / 3+(2-(4 / 3))(2 / 5)=8 / 5$. From the information, we find that $\Lambda \approx 0.7779234866$, $\Omega \approx 5.215399915$, and $\Omega_{1} \approx 0.4643521842$.

It is obvious that $F$ is an $L^{1}$-Carathéodory multi-valued map. In fact, we get $\|F(t, x)\|_{\mathcal{P}} \leq$ $(7 /(7 t+209))\left(x^{2}+1\right)$. By setting $p(t)=7 /(7 t+209), \psi(x)=x^{2}+1$, we obtain $\|p\|=1 / 30$, and there exists a number $M \in(0.3686655581,2.712485383)$ which satisfies inequality (3.2) in Theorem 3.1. Therefore, all the assumptions of Theorem 3.1 are fulfilled. Thus the Hilfer boundary value problem (3.6)-(3.7) has at least one solution on interval [1/7,13/7].

\section{Conclusion}

This paper discussed a new class of boundary value problems for sequential fractional differential equations and inclusions involving Hilfer fractional derivatives, supplemented with Riemann-Stieltjes integral multi-strip boundary conditions. Existence and uniqueness results are established in the single-valued case by using the classical Banach and Krasnosel'skii fixed point theorems and the nonlinear alternative of Leray-Schauder type. In the multi-valued case, an existence result is proved by using nonlinear alternative for contractive maps. By suitable numerical examples we verified the derived analysis. The results of the present paper are new and significantly contribute to the existing literature on the topic.

\section{Acknowledgements}

The authors thank the reviewers for their useful suggestions which improved the final version of the manuscript.

\section{Funding}

This research was funded by King Mongkut's University of Technology North Bangkok. Contract no. KMUTNB-61-KNOW-032.

Availability of data and materials

Data sharing not applicable to this article as no data sets were generated or analyzed during the current study.

Competing interests

The authors declare that they have no competing interests.

Authors' contributions

All authors contributed equally to this work. All authors read and approved the final manuscript.

\section{Author details}

'Thai-German Pre-engineering School, College of Industrial Technology, King Mongkut's University of Technology North Bangkok, Bangkok 10800, Thailand. ²Department of Mathematics, University of Ioannina, 45110 loannina, Greece. ${ }^{3}$ Nonlinear Analysis and Applied Mathematics (NAAM)-Research Group, Department of Mathematices, Faculty of Science, King Abdulaziz University, P.O. Box 80203, Jeddah 21589, Saudi Arabia. ${ }^{4}$ Department of Mathematics, Miyaneh Branch, Islamic Azad University, Miyaneh, Iran. ${ }^{5}$ Intelligent and Nonlinear Dynamic Innovations Research Center, Department of Mathematics, Faculty of Applied Science, King Mongkut's University of Technology North Bangkok, Bangkok 10800, Thailand.

\section{Publisher's Note}

Springer Nature remains neutral with regard to jurisdictional claims in published maps and institutional affiliations. 


\section{References}

1. Diethelm, K.: The Analysis of Fractional Differential Equations. Lecture Notes in Mathematics. Springer, New York (2010)

2. Kilbas, A.A., Srivastava, H.M., Trujillo, J.J.: Theory and Applications of the Fractional Differential Equations. North-Holland Mathematics Studies, vol. 204 (2006)

3. Lakshmikantham, V., Leela, S., Devi, J.V.: Theory of Fractional Dynamic Systems. Cambridge Scientific Publishers (2009)

4. Miller, K.S., Ross, B.: An Introduction to the Fractional Calculus and Differential Equations. Wiley, New York (1993)

5. Podlubny, I.: Fractional Differential Equations. Academic Press, New York (1999)

6. Samko, S.G., Kilbas, A.A., Marichev, O.I.: Fractional Integrals and Derivatives. Gordon \& Breach, Yverdon (1993)

7. Ahmad, B., Alsaedi, A., Ntouyas, S.K., Tariboon, J.: Hadamard-Type Fractional Differential Equations, Inclusions and Inequalities. Springer, Cham (2017)

8. Zhou, Y.: Basic Theory of Fractional Differential Equations. World Scientific, Singapore (2014)

9. Hilfer, R. (ed.): Applications of Fractional Calculus in Physics World Scientific, Singapore (2000)

10. Soong, T.T.: Random Differential Equations in Science and Engineering. Academic Press, New York (1973)

11. Kavitha, K., Vijayakumar, V., Udhayakumar, R., Nisar, K.S.: Results on the existence of Hilfer fractional neutral evolution equations with infinite delay via measures of noncompactness. Math. Methods Appl. Sci. 44(2), 1438-1455 (2021)

12. Subashini, R., Jothimani, K., Nisar, K.S., Ravichandran, C.: New results on nonlocal functional integro-differential equations via Hilfer fractional derivative. Alex. Eng. J. 59, 2891-2899 (2020)

13. Hilfer, R.: Experimental evidence for fractional time evolution in glass forming materials. J. Chem. Phys. 284, 399 (2002)

14. Hilfer, R., Luchko, Y., Tomovski, Z.: Operational method for the solution of fractional differential equations with generalized Riemann-Liouville fractional derivatives. Fract. Calc. Appl. Anal. 12, 299-318 (2009)

15. Furati, K.M., Kassim, N.D., Tatar, N.E.: Existence and uniqueness for a problem involving Hilfer fractional derivative. Comput. Math. Appl. 64, 1616-1626 (2012)

16. Gu, H., Trujillo, J.J.: Existence of mild solution for evolution equation with Hilfer fractional derivative. Appl. Math. Comput. 257, 344-354 (2015)

17. Wang, J., Zhang, Y.: Nonlocal initial value problems for differential equations with Hilfer fractional derivative. Appl. Math. Comput. 266, 850-859 (2015)

18. Feng, M., Zhang, X., Ge, W.: Existence theorems for a second order nonlinear differential equation with nonlocal boundary conditions and their applications. J. Appl. Math. Comput. 33, 137-153 (2010)

19. Zheng, L., Zhang, X.: Modeling and Analysis of Modern Fluid Problems. Mathematics in Science and Engineering Elsevier, London (2017)

20. Nicoud, F., Schfonfeld, T.: Integral boundary conditions for unsteady biomedical CFD applications. Int. J. Numer. Methods Fluids 40, 457-465 (2002)

21. Yusufoglu, E., Turhan, I.: A mixed boundary value problem in orthotropic strip containing a crack. J. Franklin Inst. 349, 2750-2769 (2012)

22. Renterghem, T.V., Botteldooren, D., Verheyen, K.: Road traffic noise shielding by vegetation belts of limited depth. J. Sound Vib. 331, 2404-2425 (2012)

23. Asawasamrit, S., Kijjathanakorn, A., Ntouyas, S.K., Tariboon, J.: Nonlocal boundary value problems for Hilfer fractional differential equations. Bull. Korean Math. Soc. 55, 1639-1657 (2018)

24. Wongcharoen, A., Ntouyas, S.K., Tariboon, J.: Nonlocal boundary value problems for Hilfer type pantograph fractional differential equations and inclusions. Adv. Differ. Equ. 2020, 279 (2020)

25. Nuchpong, C., Ntouyas, S.K., Tariboon, J.: Boundary value problems for Hilfer type fractional integro-differential equations and inclusions with nonlocal integro-multipoint boundary conditions. Open Math. 18, 1879-1894 (2020)

26. Sudsutad, W., Thaiprayoon, C., Ntouyas, S.K.: Existence and stability results for $\psi$-Hilfer fractional integro-differential equation with mixed nonlocal boundary conditions. AIMS Math. 6(4), 4119-4141 (2021)

27. Phuangthong, N., Ntouyas, S.K., Tariboon, J., Nonlaopon, K.: Nonlocal sequential boundary value problems for Hilfer type fractional integro-differential equations and inclusions. Mathematics 9,615 (2021)

28. Krasnosel'skiĬ, M.A.: Two remarks on the method of successive approximations. Usp. Mat. Nauk 10, $123-127$ (1955)

29. Granas, A., Dugundji, J.: Fixed Point Theory. Springer, New York (2003)

30. Deimling, K.: Multivalued Differential Equations. de Gruyter, Berlin (1992)

31. Hu, Sh., Papageorgiou, N.: Handbook of Multivalued Analysis, Volume I: Theory. Kluwer, Dordrecht (1997)

32. Smirnov, G.V.: Introduction to the Theory of Differential Inclusions. Am. Math. Soc., Providence (2002)

33. Petryshyn, W.V., Fitzpatric, P.M.: A degree theory, fixed point theorems, and mapping theorems for multivalued noncompact maps. Trans. Am. Math. Soc. 194, 1-25 (1974)

34. Lasota, A., Opial, Z.: An application of the Kakutani-Ky Fan theorem in the theory of ordinary differential equations. Bull. Acad. Pol. Sci., Sér. Sci. Math. Astron. Phys. 13, 781-786 (1965) 